PROCEEDINGS OF THE

AMERICAN MATHEMATICAL SOCIETY

Volume 131, Number 8, Pages 2577-2583

S 0002-9939(03)06885-0

Article electronically published on February 26, 2003

\title{
ON THE FUNDAMENTAL GROUP OF MANIFOLDS WITH ALMOST NONNEGATIVE RICCI CURVATURE
}

\author{
SEONG-HUN PAENG
}

(Communicated by Wolfgang Ziller)

\begin{abstract}
Gromov conjectured that the fundamental group of a manifold with almost nonnegative Ricci curvature is almost nilpotent. This conjecture is proved under the additional assumption on the conjugate radius. We show that there exists a nilpotent subgroup of finite index depending on a lower bound of the conjugate radius.
\end{abstract}

\section{INTRODUCTION}

Gromov conjectured that if a manifold has almost nonnegative Ricci curvature, then the fundamental group is almost nilpotent. Fukaya and Yamaguchi proved this conjecture when the manifold has almost nonnegative sectional curvature [FY]. Recall that a group is almost nilpotent if and only if it has a nilpotent subgroup of finite index. In $[\mathrm{FY}]$, they conjectured that if $M$ is an almost nonnegatively curved manifold, then there exists a nilpotent subgroup $\Gamma$ of $\pi_{1}(M)$ such that $\left[\pi_{1}(M): \Gamma\right] \leq \omega(n)$, where $\omega(n)$ is a constant depending only on $n$.

For a manifold with almost nonnegative Ricci curvature, if its conjugate radius is bounded below, we have weak coordinates which have a bounded weak $C^{0,{ }^{\alpha}}$-norm PWY. Using the smoothing procedure of Petersen, Wei and Ye PWY, we can apply the fibration theorem of Fukaya $[\mathrm{F}]$. Then the splitting theorem of Cheeger and Colding $[\mathrm{CC}]$ implies Gromov's conjecture immediately under the assumption on the conjugate radius.

We have the following result:

Theorem 1.1. Let $M$ be an $n$-dimensional compact Riemannian manifold with $\operatorname{diam}(M) \leq 1$ and $\operatorname{conj}_{M} \geq c_{0}>0$. Then there exist an $\epsilon>0$ and an $\omega>0$ both depending only on $n$ and $c_{0}$ such that if $\operatorname{Ric}_{M} \geq-(n-1) \epsilon^{2}$, there exists a nilpotent subgroup $\Lambda$ of $\pi_{1}(M)$ satisfying $\left[\pi_{1}(M): \Lambda\right] \leq \omega$.

We briefly review the definition of weak coordinate and weak norm. The (weak) norm of an $n$-dimensional Riemannian manifold $(M, g)$ on scale $r>0$ is defined in [PWY] as follows, where $g$ is the metric of $M$.

Received by the editors October 16, 2000 and, in revised form, August 23, 2001.

2000 Mathematics Subject Classification. Primary 53C20.

Key words and phrases. Almost nilpotent group, almost nonnegative Ricci curvature.

This work was partially supported by KIAS and by grant No.1999-2-102-002-3 from the interdisciplinary research program of the KOSEF. 
Definition 1.2. The $C^{k, \alpha}$-norm of an $n$-dimensional Riemannian manifold $(M, g)$ on scale $r>0$, denoted by $\|(M, g)\|_{C^{k, \alpha}, r}$, is defined to be the infimum of positive numbers $Q$ such that there exist imbeddings

$$
\phi_{\tau}: B(0, r) \subset \mathbb{R}^{n} \rightarrow U_{\tau} \subset M
$$

with images $U_{\tau}, \tau \in I$ (an index set), with $\rightarrow$ having the following properties:

1) $e^{-2 Q} \delta \leq \phi_{\tau}^{*}(g) \leq e^{2 Q} \delta$, where $\delta$ is the Euclidean metric,

2) every metric ball $B\left(p, \frac{r}{10} e^{-Q}\right)$ for $p \in M$ lies in some $U_{\tau}$,

3) $r^{|l|+\alpha}|| \partial^{l} g_{\tau, i j} \|_{C^{0, \alpha}} \leq Q$ for all multi-indices $l$ with $0 \leq|l| \leq k$, where $g_{\tau}=\phi_{\tau}^{*} g$.

The weak norm $\|(M, g)\|_{C^{k, \alpha}, r}^{W}$ is identically defined except that $\phi_{\tau}$ is assumed to be a local diffeomorphism instead of a diffeomorphism. Then we may regard $\phi_{\tau}$ as a weak coordinate chart. If we consider only the case that weak coordinate charts are harmonic, we call such a norm a weak harmonic norm. In [PWY], they generalized almost flat manifolds using the bounded weak $C^{0, \alpha}$-harmonic norm. We also have a generalized almost flat manifold using the bounded weak $C^{0, \alpha}$-norm (without harmonicity assumption) $[\mathrm{Pa}$. For a positive function $Q(r)$ satisfying $Q(r) \rightarrow 0$ as $r \rightarrow 0$ and $\alpha>0$, we define the following class of $n$-dimensional complete Riemannian manifolds without any assumption on the harmonicity:

$$
\mathcal{M}(n, \alpha, Q)=\left\{(M, g) \mid\|(M, g)\|_{C^{0, \alpha}, r}^{W} \leq Q(r) \text { for } 0<r \leq 1\right\} .
$$

Theorem $1.3([\mathrm{~Pa}])$. There exists an $\epsilon(n, \alpha, Q)>0$ depending on $n, \alpha$ and $Q$ such that if $M \in \mathcal{M}(n, \alpha, Q)$ and $\operatorname{diam}(M) \leq \epsilon(n, \alpha, Q)$, then a finite covering space of $M$ is a nilmanifold.

As an immediate corollary, we obtain that

Corollary 1.4. Let $M$ be an n-dimensional compact Riemannian manifold with $\operatorname{Ric}_{M} \geq-1$ and $\operatorname{conj}_{M} \geq c_{0}$. Then there exists an $\epsilon\left(n, c_{0}\right)>0$ such that if $\operatorname{diam}(M) \leq \epsilon$, then $M$ is diffeomorphic to a nilmanifold up to finite cover.

Using the smoothness of small balls as we see in Corollary 1.4, we obtain Theorem 1.1.

We would like to express our gratitude to Professor Hong-Jong Kim for his kind advice.

\section{Splitting theorem And SOlvability of $\pi_{1}(M)$}

In [FY], the authors proved the following generalized Bieberbach theorem, which is useful to find a nilpotent subgroup and a bound on the index of the nilpotent subgroup:

Theorem 2.1 ([FY] ). Let $G$ be a closed subgroup of the group of isometries of $\mathbb{R}^{n}$. Then $\pi_{0}(G)$ contains a finite index, free Abelian subgroup whose rank is not greater than $\operatorname{dim}\left(\mathbb{R}^{n} / G\right)$.

Corollary 2.2 ([FY]). Suppose, in addition, that the quotient space $\mathbb{R}^{n} / G$ is compact. Then there exists a normal subgroup $G^{\prime}$ of $G$ such that

(1) $\left[G: G^{\prime}\right]<w_{n}$, where $w_{n}$ is a number depending only on $n$,

(2) $\mathbb{R}^{n} / G^{\prime}$ is isometric to a flat torus. 
Let $M_{i}$ be a sequence of compact Riemannian manifolds with $\operatorname{Ric}_{M_{i}} \geq$ $-(n-1) \epsilon^{2} \rightarrow 0, \operatorname{conj}_{M} \geq c_{0}$ and $\operatorname{diam}(M) \leq 1$. We denote $\pi_{1}\left(M_{i}\right)$ by $\Pi_{i}$. From [CC], we have the following convergence with respect to the pointed GromovHausdorff distance:

$$
\left(\tilde{M}_{i}, \Pi_{i}\right) \rightarrow\left(\mathbb{R}^{k} \times X, \Pi\right)
$$

for some compact length space $X$ and an isometry group $\Pi$ of $X$. Also $\gamma \in \Pi$ is of the form $\left(\gamma_{1}, \gamma_{2}\right)(x, y)=\left(\gamma_{1}(x), \gamma_{2}(y)\right)$, where $\gamma_{1}$ and $\gamma_{2}$ are isometries on $\mathbb{R}^{k}$ and $X$, respectively [CG].

Now we review the construction of solvable subgroups with a bounded index. From Corollary 2.2, if we consider a finite $(\leq w(k))$ covering space of $M_{i}$, we may assume that $M_{i}$ converges to a fiber bundle $X_{1}$ over $T^{k_{1}}$, where $k_{1} \leq k$ and $k_{1}$ is the rank of the maximal discrete Abelian subgroup of $\Pi$. So we may assume that $M_{i} \rightarrow X_{1}$. Then $\gamma_{1}$ can be decomposed to $\left(\gamma_{11}, \gamma_{12}\right)$, where $\gamma_{11}$ and $\gamma_{12}$ are isometries on $\mathbb{R}^{k_{1}}$ and $\mathbb{R}^{k-k_{1}}$, respectively. We may consider $\gamma_{11}$ as an element of $\pi_{1}\left(T^{k_{1}}\right)$. Let $p_{1}(\gamma)$ be $\gamma_{11}$ and $p_{2}(\gamma)$ be $\left(\gamma_{12}, \gamma_{2}\right)$. We denote $\operatorname{Ker}\left(p_{1}\right)$ by $K$. Then $K$ is normal subgroup of $\Pi$ and $\Pi / K \simeq \mathbb{Z}^{k_{1}}$. Using Theorem 3.10 in [FY], we can find a normal subgroup $K_{i}$ of $\Pi_{i}$ such that

$$
\left(\tilde{M}_{i}, K_{i}\right) \rightarrow\left(\mathbb{R}^{k} \times X, K\right)
$$

and

$$
\Pi_{i} / K_{i} \simeq \Pi / K=\mathbb{Z}^{k_{1}} .
$$

Using Theorem 1.3 or Corollary 1.4 from the previous section, we can find $\delta\left(n, c_{0}\right)>0$ such that $\pi_{1}\left(\phi_{i}(B(0, \delta))\right)$ is almost nilpotent, so there exists a nilpotent subgroup $\tilde{H}_{i}$ such that

$$
\left[\pi_{1}\left(\phi_{i}(B(0, \delta))\right): \tilde{H}_{i}\right] \leq \kappa(n)
$$

for some constant $\kappa$ depending only on $n$, where $\phi_{i}$ is a weak coordinate of $M_{i}$ (see $[\mathrm{G}]$ and $[\mathrm{Pa}])$. Let $i: \phi_{i}(B(0, \delta)) \rightarrow M_{i}$ be the inclusion map. We denote $i_{*}\left(\tilde{H}_{i}\right)$ by $H_{i}$. Let

$$
\left(\tilde{M}_{i}, H_{i}\right) \rightarrow\left(\mathbb{R}^{k} \times X, H\right)
$$

for some isometry group $H$ of $\mathbb{R}^{k} \times X$. If we take $\delta>0$ sufficiently small, we may assume that $p_{1}(H)=\mathrm{Id}$, so $H \subset K$ and $H_{i} \subset i_{*}\left(\pi_{1}\left(\phi_{i}(B(0, \delta))\right)\right) \subset K_{i}$. From Definition $1.2, \phi_{i}(B(0, \delta))$ contains an $\epsilon$-ball for a constant $\epsilon\left(n, c_{0}\right)>0$ depending on $n$ and $c_{0}$.

Using the Bishop-Gromov comparison theorem, we obtain that for any $x \in \tilde{M}_{i}$,

$$
\frac{\operatorname{vol}(B(x, 20))}{\operatorname{vol}(B(x, \epsilon / 100))} \leq C_{1}\left(n, c_{0}\right)
$$

for some constant $C_{1}\left(n, c_{0}\right)>0$ depending only on $n$ and $c_{0}$. (In fact, $C_{1}$ depends on $\epsilon$ but $\epsilon$ is determined by $c_{0}$.) For $y \in B(x, 10)$, we have

$$
\frac{\operatorname{vol}(B(x, 10))}{\operatorname{vol}(B(y, \epsilon / 100))} \leq \frac{\operatorname{vol}(B(y, 20))}{\operatorname{vol}(B(y, \epsilon / 100))} \leq C_{1}\left(n, c_{0}\right),
$$


so the maximal number of $\epsilon$-balls disjointly contained in a 10-ball is bounded by $C_{1}$. For convenience, if we assume that $\operatorname{diam}(X) \leq 20$,

$$
X=\bigcup_{i=1}^{N} B\left(y_{i}, \epsilon / 10\right)
$$

for $N \leq C_{1}$. Then we obtain that

$$
\begin{aligned}
{\left[K_{i}: H_{i}\right] } & =\left[K_{i}: i_{*}\left(\pi_{1}\left(\phi_{i}(B(0, \delta))\right)\right)\right]\left[i_{*}\left(\pi_{1}\left(\phi_{i}(B(0, \delta))\right)\right): H_{i}\right] \\
& \leq \kappa(n) C_{1}\left(n, c_{0}\right) .
\end{aligned}
$$

We choose $\left\{\alpha_{1}^{(i)}, \cdots, \alpha_{k_{1}}^{(i)}\right\} \subset \Pi_{i}$ such that $\alpha_{j}^{(i)} \rightarrow \alpha_{j} \in \Pi$ as $i \rightarrow \infty$ and $\left\{p_{1}\left(\alpha_{j}\right)\right\}$ is a basis of $\pi_{1}\left(T^{k_{1}}\right)$, where $T^{k_{1}}$ is mentioned above. Let $A(\alpha)$ be an automorphism on $K_{i}$ defined by

$$
A(\alpha)(\gamma)=\alpha \gamma \alpha^{-1}
$$

From $(2.1)$, we can find $l \leq\left(\kappa(n) C_{1}\left(n, c_{0}\right)\right)^{k_{1}}$ such that

$$
A\left(\alpha_{j}^{(i)}\right)^{l}\left(H_{i}\right)=\left(\alpha_{j}^{(i)}\right)^{l} H_{i}\left(\alpha_{j}^{(i)}\right)^{-l}=H_{i} .
$$

We denote $\left(\alpha_{j}^{(i)}\right)^{l}$ by $\beta_{j}^{(i)}$. Let $\Gamma_{i}$ be the subgroup generated by $K_{i}$ and $\left\{\beta_{j}^{(i)} \mid j=\right.$ $\left.1, \cdots, k_{1}\right\}$. From $(2.2), H_{i}$ is a normal subgroup of $\Gamma_{i}$ and

$$
\begin{aligned}
{\left[\Pi_{i}: \Gamma_{i}\right] } & =\left[K_{i}: H_{i}\right]\left[\left\langle\alpha_{1}^{(i)}, \cdots, \alpha_{k_{1}}^{(i)}\right\rangle:\left\langle\beta_{1}^{(i)}, \cdots, \beta_{k_{1}}^{(i)}\right\rangle\right] \\
& \leq l^{k_{1}+1} \leq\left(\kappa(n) C_{1}\left(n, c_{0}\right)\right)^{2 n^{2}} .
\end{aligned}
$$

We have

$$
1 \rightarrow K_{i} \rightarrow \Gamma_{i} \rightarrow \mathbb{Z}^{k_{1}} \rightarrow 1
$$

and

$$
1 \rightarrow K_{i} / H_{i} \rightarrow \Gamma_{i} / H_{i} \rightarrow \mathbb{Z}^{k_{1}} \rightarrow 1
$$

Applying Lemma 4.4 in [FY], we obtain that

$$
1 \rightarrow \mathbb{Z}^{k_{1}} \rightarrow \Gamma_{i} / H_{i} \rightarrow \Omega \rightarrow 1
$$

for some finite group $\Omega$ such that $|\Omega| \leq C_{2}(n)$ for some constant $C_{2}$ depending only on $n$. Hence we can find a solvable subgroup $\Gamma_{i}^{\prime}$ such that

$$
\Gamma_{i}^{\prime} / H_{i} \simeq \mathbb{Z}^{k_{1}}
$$

and

$$
\left[\Pi_{i}: \Gamma_{i}^{\prime}\right]=\left[\Pi_{i}: \Gamma_{i}\right]\left[\Gamma_{i}: \Gamma_{i}^{\prime}\right] \leq C_{3}(n):=\left(\kappa(n) C_{1}\left(n, c_{0}\right)\right)^{2 n^{2}} C_{2}(n)<\infty .
$$

From this, the boundedness of indices of solvable subgroups of $\pi_{1}(M)$ is obtained.

\section{Proof of Theorem 1.1}

We will find nilpotent subgroups $\Lambda_{i} \subset \Gamma_{i}^{\prime}$ such that $\left[\Pi_{i}: \Lambda_{i}\right]<\infty$. Since $\tilde{M}_{i} \rightarrow \mathbb{R}^{k} \times X$, we have a pointed Hausdorff approximation

$$
f_{i}: B\left(o_{i}, 1 / \epsilon_{i}\right) \subset \tilde{M}_{i} \rightarrow \mathbb{R}^{k} \times X,
$$

as $\epsilon_{i} \rightarrow 0$. For the precise definition of Hausdorff approximation, see [FY]. Assume that $f_{i}\left(o_{i}\right)$ converges to $o=\left(o_{1}, o_{2}\right) \in \mathbb{R}^{k} \times X$. Let $N$ be $\left\{\left(x, o_{2}\right) \mid x \in \mathbb{R}^{k}\right\} \subset \mathbb{R}^{k} \times X$ and $N_{i}$ be $f_{i}^{-1}(N)$.

As in the previous section, we have a quotient map $q_{i}: \Gamma_{i}^{\prime} \rightarrow \mathbb{Z}^{k_{1}}$. We take a subset $B_{i}=\left\{\gamma_{1}^{(i)}, \cdots, \gamma_{k_{1}}^{(i)}\right\}$ of $\Gamma_{i}^{\prime}$ such that $q_{i}\left(B_{i}\right)$ is a basis of $\mathbb{Z}^{k_{1}}$. Since $\gamma_{j}^{(i)}$ 
converges to an isometry $\gamma_{j}=\left(p_{1}\left(\gamma_{j}\right), p_{2}\left(\gamma_{j}\right)\right)$ of the $\mathbb{R}^{k} \times X$, there exist $l_{j} \leq C_{1}$ such that

$$
d\left(\gamma_{j}^{l_{j}}(o), N\right) \leq \epsilon
$$

so

$$
d\left(\left(\gamma_{j}^{(i)}\right)^{l_{j}}\left(o_{i}\right), N_{i}\right) \leq 2 \epsilon
$$

for $C_{1}$ and $\epsilon$ in section 2. We denote $\gamma_{j}^{l_{j}}$ and $\left(\gamma_{j}^{(i)}\right)^{l_{j}}$ by $\gamma_{j}^{\prime}$ and $\gamma_{j}^{\prime(i)}$, respectively. Let $x_{j}$ be $p_{1}\left(\gamma_{j}^{\prime}(o)\right)$. We take a point $x_{j}^{(i)}$ in $f_{i}^{-1}\left(x_{j}\right)$. (It is possible that $f_{i}^{-1}\left(x_{j}\right)=\emptyset$. But we can find a sequence $x_{j, h} \rightarrow x_{j}$ as $h \rightarrow \infty$ in $\mathbb{R}^{k} \times X$ such that $x_{j, h} \in \operatorname{Im}\left(f_{i}\right)$. So we may assume its existence.)

In Definition 1.2, a weak coordinate for an $r$-ball is used. Now we use a weak coordinate-like map for the tubular neighborhood of geodesic segment from $o_{i}$ to $x_{j}^{(i)}$. We consider a weak coordinate-like map by the same method as in Definition 1.2: We denote $d\left(\gamma_{j}(o), o\right)$ by $s_{j}$. Let $c_{j}^{(i)}(t)$ be the shortest geodesic segment from $o_{i}$ to $x_{j}^{(i)}$. We may assume that $c_{j}^{(i)}(t)$ is a unit speed curve. On $T_{\delta}=$ $\left(-10 \delta, s_{j}+10 \delta\right) \times B(0,10 \delta)$, we define $\psi_{i}$ as follows:

$$
\begin{aligned}
\psi_{i}: T_{\delta} & \rightarrow \tilde{M}_{i} \\
(t, y) & \mapsto \exp _{c_{j}^{(i)}(t)} y
\end{aligned}
$$

where $B(0, R)$ is the $R$-ball centered at 0 in $\mathbb{R}^{n-1}$ and $\delta$ is the positive constant in section 2. Then $\psi_{i}$ is a nonsingular map. We consider a metric $\psi_{i}^{*} g_{i}$ on $T_{\delta}$, where $g_{i}$ is the metric of $M_{i}$. From [AC], $\left\{\left(T_{\delta}, \psi_{i}^{*} g_{i}\right)\right\}$ is $C^{0, \alpha}$-compact. So $T_{\delta}$ has a $C^{0, \alpha}$-bounded norm. Then there exists a weak coordinate-like map

$$
\phi_{i}: T_{\delta} \rightarrow \tilde{M}_{i}
$$

satisfying the conditions in Definition 1.2. Using the compactness result of [AC], we know that $\left(T_{\delta}, \phi_{i}^{*} g_{i}\right)$ converges to a $C^{0, \alpha}$-Riemannian manifold. By the fibration theorem in $[\mathrm{F}]$ and $[\mathrm{W}]$, there exists an almost Riemannian submersion

$$
\Psi_{i}: T_{\delta} \rightarrow I
$$

where $I=\left(-10 \delta, s_{j}+10 \delta\right)$. Then we have the following convergence:

$$
\left(T_{\delta}, \phi_{i}^{*} g_{i}\right) \rightarrow\left(T, g_{0}\right) \subset\left(I \times Y, g_{0}\right),
$$

where $Y \subset \mathbb{R}^{n-1}$ has a $C^{0, \alpha}$-Riemannian metric and $g_{0}$ is an almost product metric of the metric on $I \subset \mathbb{R}$ and the metric on $Y$. There exists a diffeomorphism

$$
\Phi_{i}: T \rightarrow T_{\delta}
$$

such that

$$
\left\|\Phi_{i}^{*}\left(\phi_{i}^{*} g_{i}\right)-g_{0}\right\| \rightarrow 0
$$

in $C^{0, \alpha}$-topology. From $[\mathrm{CH}]$, we know that isometries converge in $C^{1, \alpha}$-topology. Isometries from a small ball $B\left(o_{i}, \delta\right) \subset \tilde{M}_{i}$ to $\operatorname{Im}\left(\phi_{i}\right) \subset \tilde{M}_{i}$ can be lifted to isometries of a $\delta$-ball in $T_{\delta}$. We denote the lifting of $\alpha_{i} \in \operatorname{Isom}\left(M_{i}\right)$ by $\tilde{\alpha}_{i} \in \operatorname{Isom}\left(T_{\delta}\right)$. Then any elements $\gamma^{(i)} \in \pi_{1}\left(\phi_{i}(B(0, \delta))\right)$ can be lifted to an isometry $\tilde{\gamma}^{(i)}$ on a $\delta$-ball in $T_{\delta}$, where $\phi_{i}(0)=o_{i}$. 
If $\gamma^{(i)} \in \tilde{H}_{i}$, then an almost isometric map $\Phi_{i}^{-1} \gamma^{(i)} \Phi_{i}: T \subset \mathbb{R}^{n} \rightarrow T \subset \mathbb{R}^{n}$ can be written in the following form: We denote by $\tau(x)$ a map $f$ such that $f \in C^{1}$ and $f(0)=D f(0)=0$. Then we have

$$
G(x)=\Phi_{i}^{-1} \gamma^{(i)} \Phi_{i}(x)=b+B x+\tau\left(d\left(x, \Psi_{i}(x)\right)\right),
$$

where $b=G(0)$ and $\|B-\mathrm{I}\| \leq \epsilon_{1}\|b\|$ for some small $\epsilon_{1}>0$ since it is an isometry on $B\left(o_{i}, \delta\right)$ which is almost flat by $[\mathrm{Pa}$ and $[\mathrm{PWY}]$.

We define $F_{j}$ as follows:

$$
F_{j}=\Phi_{i}^{-1} \gamma_{j}^{(i)} \Phi_{i}
$$

and write $F_{j}$ as follows:

$$
F_{j}(x)=a_{j}+A_{j} x+\tau(x),
$$

where $a_{j}=F_{j}(0)$. Similarly as section 2 , we denote $F_{j} G F_{j}^{-1}$ by $A\left(F_{j}\right)(G)$. Then we also obtain that for $\|x\| \leq 10 b$,

$$
A\left(F_{j}\right)(G)(0)=\left(\mathrm{I}-A_{j} B A_{j}^{-1}\right) a_{j}+A_{j} b+\tau(b) .
$$

Since $A_{j}$ is an almost isometry and $\|B-I\| \leq \epsilon_{1}\|b\|$, we have $d\left(A\left(F_{j}\right)(G)(o), A_{j} b\right) \leq$ $10 \epsilon_{1}\left\|a_{j}|\|||b| \mid\right.$. The cardinality of $\left\{\gamma \in H_{i} \mid b-\delta \leq d(\gamma(o), o) \leq b+\delta\right\}$ is bounded by $2^{k_{1}}$ by the choice of a nilpotent group $H_{i}$. Considering the orbit $\left\{A\left(F_{j}^{l}\right)(G)(0) \mid l=\right.$ $1,2, \cdots\}$, we have $\left\|A_{j}^{s}-\mathrm{I}\right\| \leq \epsilon_{1}\left\|a_{j}\right\|$ for $s \leq 2^{k_{1}} \leq 2^{n}$. So we obtain that

$$
\left\|A\left(F_{j}^{s}\right)(G)(0)-G(0)\right\| \leq 10 \epsilon_{1} s\|a|\||| b\|
$$

and

$$
d\left(\left(\gamma_{j}^{\prime}\right)^{s} \gamma\left(\gamma_{j}^{\prime}\right)^{-s}(o), \gamma(o)\right) \leq 10 \epsilon_{1}\left\|\gamma^{s}\right\|\|\mid \gamma\|,
$$

where $\|\alpha\|=d(\alpha(o), o)$ for $\alpha \in \Pi$. From this, $\Lambda_{i}=\left\langle\left\{H_{i},\left(\gamma^{\prime}{ }_{j}^{(i)}\right)^{s} \mid j=1,2, \cdots, k_{1}\right\}\right\rangle$ is a nilpotent group since

$$
\|[\alpha, \beta]\|=d\left([\alpha, \beta]\left(o_{i}\right), o_{i}\right) \leq 10 \epsilon_{1}|| \alpha|||| \beta \|
$$

for $\alpha, \beta \in \Lambda_{i}\left[\mathrm{~Pa}\right.$. Then $\left[\Pi_{i}: \Lambda_{i}\right] \leq\left(\kappa(n) C_{1}\left(n, c_{0}\right)\right)^{2 n^{2}} C_{2}(n) C_{1}(n)^{2 n^{2}}<\infty$. Until now, we proved that for any sequence of manifolds $\left\{M_{i}\right\}$ which satisfies the condition of Theorem 1.1, there exists a subsequence $\left\{M_{i_{j}}\right\}$ such that $\pi_{1}\left(M_{i_{j}}\right)$ 's have nilpotent subgroups whose indices are bounded.

If we assume that Theorem 1.1 is false, then we can find a sequence of manifolds $\left\{M_{i}\right\}$ such that $\lim _{i \rightarrow \infty}\left[\pi_{1}\left(M_{i}\right): \Lambda_{i}\right]=\infty$ for any nilpotent subgroups $\Lambda_{i}$, which is a contradiction to the fact we have proved. This completes the proof.

\section{REFERENCES}

[AC] M. T. Anderson, J. Cheeger, $C^{\alpha}$-compactness for manifolds with Ricci curvature and injectivity radius bounded below, J. Diff. Geom. 35 (1992), 265-281. MR 93c:53028

[BK] P. Buser, H. Karcher, Gromov's almost flat manifolds, Astérisque, 1981. MR 83m:53070

[CC] J. Cheeger, T. B. Colding, Lower bounds on Ricci curvature and the almost rigidity of warped products, Ann. Math. 144 (1996), 189-237. MR 97h:53038

[CG] J. Cheeger, D. Gromoll, The splitting theorem for manifolds of nonnegative Ricci curvature, J. Diff. Geom. 6 (1971), 119-128. MR 46:2597

$[\mathrm{CH}] \quad$ E. Calabi, P. Hartman, On the smoothness of isometries, Duke Math. J. 37 (1970), 741750. MR 44:957

[F] K. Fukaya, Collapsing Riemannian manifolds to ones of lower dimensions, J. Diff. Geom. 25 (1987), 139-156. MR 88b:53050

[FY] K. Fukaya, T. Yamaguchi, The fundamental groups of almost nonnegatively curved manifolds, Ann. Math. 136 (1992), 253-333. MR 93h:53041

[G] M. Gromov, Almost flat manifolds, J. Diff. Geom. 13 (1978), 231-241. MR 80h:53041 
[Pa] S.-H. Paeng, A generalized almost flat manifolds under a bounded $C^{0, \alpha}$-weak norm, Arch. Math. (Basel) 77 (2001), 423-429. MR 2002g:53050

[PWY] P. Petersen, G. Wei, R. Ye, Controlled geometry via smoothing, Comment. Math. Helv. 74 (1999), 345-363. MR 2000h:53040

[W] G. Wei, Ricci curvature and Betti number, J. Geom. Anal 7 (1997), 377-386. MR 2000d:53062

Department of Mathematics, Konkuk University, 1 Hwayang-Dong, Gwanguin-gu, Seoul 143-701, Korea

E-mail address: shpaeng@kkucc.konkuk.ac.kr 